\title{
Two kinds of focus constructions in K'iche'*
}

\author{
Murat Yasavul \\ Ohio State University
}

\begin{abstract}
Based on original fieldwork data, I argue for a difference in meaning between two focus constructions in K'iche' (Mayan). In particular, I show that the interpretation of focus constructions with aree 'FOC' gives rise to existence and exhaustivity implications (the latter when they are used as answers), both of which do not necessarily arise from the interpretation of focus constructions without aree. I discuss how to analyze these implications and review previous approaches to similar phenomena. I also develop a dynamic account that captures the empirical generalizations I propose.
\end{abstract}

Keywords: Focus, K'iche', clefts, exhaustivity, dynamic semantics

\section{Introduction}

K'iche' is a Mayan language spoken by over a million people in the central and western highlands of Guatemala (Richards 2003). In K'iche', there is a designated focus position preceding the predicate as shown in (1). ${ }^{1}$ Expressions in this position can be preceded by the so-called focus particle aree 'FOC' as in (2).

(1) A Raul x- Ø-war-ik.

CLF Raul CMP-A3-sleep-SS

'RAUL slept.'
(2) Aree a Raul x- Ø-war-ik. FOC CLF Raul CMP-A3-sleep-SS 'It was RAUL who slept.'

This paper investigates meaning differences between the two focus constructions exemplified in (1) and (2). I show, on the basis of original fieldwork data, that the

* I thank my consultants as well as Carl Pollard, Judith Tonhauser, Jefferson Barlew, Manjuan Duan, Greg Kierstead and the Syntax-Semantics Discussion Group "Synners" at Ohio State for helpful discussions. Many of the ideas presented here grew out of long discussions with Carl Pollard and I gratefully acknowledge his insights. Of course, the usual disclaimers apply. The fieldwork for this project was supported by the Tinker Field Research and Global Gateway Research Grants as well as the Department of Linguistics and the College of Arts and Humanities at Ohio State.

1 The K'iche' data presented here come from original fieldwork in Santa María Tzejá, Ixcán, Guatemala. I use the following glosses: A1 (P), A2(P), A3(P) = absolutive first, second, third person singular (plural); E1(P), E2(P), E3(P) = ergative first, second, third person singular (plural); CLF = classifier; $\mathrm{CMP}=$ completive; $\mathrm{DET}=$ determiner; $\mathrm{DIR}=$ directional $; \mathrm{FOC}=$ focus particle $; \mathrm{GEN}=$ genitive $; \mathrm{ICP}=$ incompletive; $\mathrm{PL}=$ plural; $\mathrm{SS}=$ status suffix.

C2013 Yasavul 
interpretation of focus constructions with aree 'FOC' is different from those without it. In particular, the interpretation of the former, but not the latter, gives rise to existence and exhaustivity implications (the latter when it is used as an answer ${ }^{2}$ ) similar to it-clefts in English or preverbal focus in Hungarian (Delin 1992; É. Kiss 1998). Previously, this property of K'iche' was either overlooked (e.g., Larsen 1988; Trechsel 1993) or not explicitly shown to be the case (Can Pixabaj \& England 2011). More importantly, this finding goes against the claim that focus in Mayan has a cleft interpretation across-the-board (Aissen 1992). Rather, I argue that sentences with and without aree 'FOC' have different discourse functions in K'iche'.

The rest of the paper is organized as follows. In $\S 2$, I provide a brief overview of the relevant K'iche' data and review the previous claims about their interpretations. In $\S 3$, I discuss in detail the implications of focus constructions with aree. In $\S 4$, I provide a dynamic analysis that captures the generalizations reached in $\S 3$. In $\S 5$, I discuss the previous claims about the interpretation of related $i t$-cleft/focusmovement constructions. I conclude in $\$ 6$ with a brief discussion.

\section{Background on K'iche'}

K'iche' has an ergative-absolutive agreement system and a predicate-initial basic word order (Larsen 1988; England 1991). As in other Mayan languages, it has a designated pre-predicate position for focused expressions (Larsen 1988; England 1991) as exemplified in (3). I will refer to such constructions as answer focus constructions, or answer FC for short, when they are used to answer overt questions.

(3) Context: Who slept?

A Raul x- $\emptyset$-war-ik.

CLF Raul CMP-A3-sleep-ss

'RAUL slept.'

The constituents occupying the focus position are generally understood to be semantically "prominent" in some sense (Larsen 1988) which is reflected in the cleft/pseudo-cleft translations into English, a standard practice in the Mayan literature $^{3}$ (e.g., Larsen 1988; Aissen 1992; Trechsel 1993). A common assumption about focus constructions has been that they are interpreted like clefts in that they are associated with existence and uniqueness implications (Aissen 1992; Trechsel 1993). Aissen (1992: 49-50), for example, claims that focus constructions in Mayan have a cleft interpretation by drawing on data from Tzotzil. She argues that focus

2 I use the term answer for sentences or sentence fragments which are anaphoric to overt questions. I do not assume that every declarative sentence is an answer to an overt or otherwise inferred question as in Roberts 1996/2012.

3 The reason why I do not follow this tradition for sentences like (3) will become clear in $\S 3$. 
constructions are associated with an existence presupposition which projects, that is, remains an utterance implication, when such sentences are negated or turned into interrogatives. She also argues that the denotation of the focused expression is asserted to be the unique entity that satisfies this presupposition. Similarly, Trechsel (1993: 42) claims that, like their translations, focus constructions in K'iche' have an existence presupposition, and follows Aissen in claiming that such sentences assert that the entity denoted by the focused expression is the only entity in the domain of discourse that has the property in question.

However, the claims about (i) the implications of focus constructions and (ii) their behavior when embedded under entailment-canceling operators seem to be based on English translations. In other words, the Mayan focus constructions themselves are not shown to have these implications with the alleged behavior. As Matthewson (2004: 389) argues, translations do not provide direct evidence for meaning. Rather, she argues that reliable evidence for meaning can be obtained from judgments by native speakers elicited for sentences uttered in particular contexts. Therefore, whether focus constructions have the implications mentioned above and whether some of these implications project under entailment-canceling operators ought to be determined based on native speaker judgments elicited in properly constructed contexts (Matthewson 2004; Tonhauser, Beaver, Roberts \& Simons 2013).

K'iche' has another focus construction, alongside the one in (3), where focused expressions are preceded by the so-called focus particle aree $^{4}$ 'FOC' as in (4a), and where focused expressions have to occur before the predicate, given $(4 b, c) .{ }^{5}$ I will refer to such constructions as aree focus constructions, or aree FC for short. ${ }^{6}$

(4) a. Aree a Raul x- $\emptyset$-war-ik. FOC CLF Raul CMP-A3-sleep-SS

'It is RAUL who slept.'

$$
\begin{aligned}
& \text { b.?Aree X- } \emptyset \text {-war a Raul. } \\
& \text { FOC CMP-A3-sleep CLF Raul } \\
& \text { c.*X- } \emptyset \text {-war aree a Raul. } \\
& \text { CMP-A3-sleep FOC CLF Raul }
\end{aligned}
$$

4 As aree can also be used as a copula as in (i), K'iche' seems to behave like those languages where the morphological focus marker is cognate with the copula in the language (É. Kiss 2006).

(i) A Raul aree jun tijoxel.

CLF Raul aree one student

'Raul is a student.'

5 The example in (4b) is marginal for the consultants I worked with who commented that "this is not the usual way of saying it." Such sentences where aree and the focused expression are separated get worse when there is more intervening material between the two expressions.

6 As we'll see below, this construction can also be used to answer questions but this is not a requirement. So, I reserve the term answer focus for those that do not have aree. 
Although, historically, the interpretations of the two focus constructions in K'iche' have not been distinguished (e.g., Larsen 1988; Trechsel 1993), in their recent study on K'iche' texts, Can Pixabaj \& England (2011) argue that these constructions have different interpretations. In particular, Can Pixabaj \& England (2011: 23) claim that the aree FC "operates much like English cleft in terms of usage and meaning." However, they provide no empirical evidence for this claim apart from translations. Like the previous accounts, they do not show whether, say, (4a) has implications that would justify comparing its interpretation to that of an English it-cleft. Moreover, as Matthewson (2004: 371) points out, the relevant evidence for such a claim cannot be obtained from texts, as one needs negative evidence, that is, evidence for when one cannot use a cleft-like construction. What I show in this paper is that the two focus constructions indeed differ in terms of their interpretations. In the next section, I discuss what these differences are.

\section{Implications associated with K'iche' focus constructions}

\subsection{Existence implication}

The first implication that distinguishes aree FCs from answer FCs is an existence implication which acts as a constraint on the common ground (CG). This constraint requires that there be an individual (possibly plural) with the property under discussion. In (5), for example, if the existence of an individual with the relevant property (here, having a computer) in a given domain (here, Maria's class) is not in the CG, then using aree is not felicitous as shown in (5b). Crucially, answer FCs are not subject to this requirement as shown in (5a).

(5) Context: An inspector from the Ministry of Education is visiting the community. He's surveying all the primary school teachers about their students and asking them questions like the following: Who (in your class) has a computer? Who has more than two siblings? Whose parents are divorced? Maria, a teacher, knows that in her class, Raul has a computer. When the inspector asks his first question, she says:

a. A Raul (k'o jun u-kematz'ib'). b.\#Aree a Raul (k'o jun u-kematz'ib'). CLF Raul exist one E3-computer 'Raul (has a computer).' FOC CLF Raul exist one E3-computer 'It is Raul (who has a computer).'

However, in a minimally different context where the existence implication is in the CG, say, because the principal or another teacher told the inspector about Maria's class and Maria knows that the inspector knows about this-call this context $\left(5^{\prime}\right)$ - then (5b) is felicitous alongside (5a). This shows that an answer FC like (5a) can be used whether the CG satisfies the existence implication or not, but the aree FC 
Two kinds of focus constructions in K'iche'

can only be used when it is satisfied. To show that this implication is a presupposition of aree FCs, I use a standard diagnostic called the family of sentences (FoS) tests (Chierchia \& McConnell-Ginet 1990). These tests involve checking whether an implication $m$ of a declarative sentence $S$ is projective, that is, whether it remains an utterance implication of the FoS variants of $S$ which are the negative of $S$, the interrogative of $S$, a modal variant of $S$, and a conditional with $S$ as antecedent (Tonhauser et al. 2013). The FoS variants of (5b) are judged acceptable when the $\mathrm{CG}$ entails the existence implication as in (6a-d) and unacceptable otherwise.

(6) Context: Same as $\left(5^{\prime}\right)$.

a. (Maria asks another teacher who's present and knows about her class:)

Aree a Raul k'o jun u-kematz'ib'?

FOC CLF Raul exist one E3-computer

'Is it Raul who has a computer?'

b. Xaqri aree a Raul k'o jun u-kematz'ib'.

perhaps FOC CLF Raul exist one E3-computer

'Perhaps it is Raul who has a computer.'

c. We aree a Raul k'o jun u-kematz'ib', in Ø-w-eta'm che

if FOC CLF Raul exist one E3-computer I A3-E1-know COMP

k- $\emptyset$-u-koj k- $\emptyset$-u-b'an etz'anem.

ICP-A3-E3-use ICP-A3-E3-do game

'If it's Raul who has a computer, I'm sure he uses it for playing games.'

d. (Another teacher who's present and knows about Maria's class says that Raul has a computer and Maria says:)

Aree $=t$ a Raul k'o jun u-kematz'ib'. Aree a Roberto.

FOC $=$ NEG CLF Raul exist one E3-computer FOC CLF Roberto

'It isn't Raul who has a computer. It is Roberto.'

Since the existence implication is both a projective implication and a constraint on context, I analyze it as a presupposition. Thus, I claim that a focus construction of the form Aree $X P$, where $X$ stands for the expression in the focus position and $P$ for the expression whose denotation is the property under discussion, presupposes that there is an individual (singular or plural) with property $P$. In the next section I turn to a discussion of the second property of aree FCs that can be observed when they are used as answers.

\subsection{Exhaustivity implication}

The second implication that distinguishes aree FCs from answer FCs can be called an exhaustivity implication, following much of the previous work on the interpretation 
of it-cleft/focus movement constructions, e.g., in English and Hungarian (É. Kiss 1998). For these latter constructions, it has been claimed that exhaustivity amounts to identifying the entity denoted by the focused expression as the only (or maximal) entity bearing the property under discussion (among others, Horn 1981; Szabolcsi 1981; Roberts 1998; Percus 1997; É. Kiss 1998; Krifka 2008; Büring 2011; Velleman, Beaver, Destruel, Bumford, Onea \& Coppock 2012). There is a considerable amount of discussion in the literature as to how to characterize and classify this implication, which I will discuss in detail in $§ 5$. But first I will exemplify this implication in K'iche' and explain why it arises. To illustrate, consider the data in (7), which show a contrast between the two focus constructions.

(7) Context: Raul and Roberto are talking about an exam that Raul had taken that has just been graded. He says that the teacher gave a piece of candy to those who got $100 \%$. Roberto asks (7a) and Raul says (7b). (7c) is unacceptable.

a. Chin $x-\emptyset$-r-esaj juntir utz?

who CMP-A3-E3-take.out all good

'Who got $100 \%$ ?'

b. A Juan r-ichb'il al Maria. Ø-w-eta'm taj we k'o jun chik. / CLF Juan E3-companion CLF Maria A3-E1-know NEG if exist one another

Al Juana xuquje'.

CLF Juana too

'Juan and Maria. I don't know if anyone else did./ Juana did, too.'

c. Aree a Juan r-ichb'il al Maria. \# ฤ-w-eta'm taj we k'o jun FOC CLF Juan E3-companion CLF Maria A3-E1-know NEG if exist one chik. /\# Al Juana xuquje'.

another CLF Juana too

'It was Juan and Maria. I don't know if anyone else did./ Juana did, too.'

As (7b) shows, with an answer FC it is possible for the speaker to not know whether someone else other than Juan and Maria got $100 \%$, or to use an additive particle like xuquje' 'too' to add another individual to the plurality of people who got $100 \%$. However, as (7c) shows, using an aree FC precludes such continuations. So then the question is why the two constructions behave differently. In the previous section, I argued that an aree FC gives rise to an existence presupposition, namely that the (discontinuous) expression Aree... $(P)$ is an anaphoric expression requiring that there be an individual in the context with the relevant property. I claim that the contrast we see in (7) arises because the antecedent in the context of the question in (7a) is the maximal plurality of individuals with the property of getting $100 \%$. The aree FC identifies this plurality with the denotation of the focused expression, hence the infelicity of the sentences following the aree $\mathrm{FC}$ in (7c). 
To see why the answers in (7) are about the maximal plurality of individuals who got $100 \%$, we need to understand the context in which (7b) and (7c) are uttered and, in particular, what the question they are answers to contributes to the context. To that end, I will adopt two ideas from Hamblin $(1957,1971)$ for the analysis of questions. Hamblin (1957) claims that (i) a question denotes the set of its possible answers, (ii) such possible answers are together exhaustive and (iii) mutually exclusive. Note that it is not only that a question denotes the set of its possible answers (the so-called "Hamblin alternatives"), but such answers are complete answers as they are defined to be mutually exclusive. ${ }^{7}$ In addition, Hamblin (1971: 134) defines what he calls the presumption of a question as "equivalent to the disjunction of its answers." The disjunction of the possible answers of a question is equivalent to an existential quantification on the set of its possible answers. Since possible answers are complete and mutually exclusive, the presumption of a question requires not only existence but the unique existence of a true complete answer. Thus, in an extensional setting, the Hamblin presumption (HP) of a question is true at a given world $w$ iff exactly one of the propositions in its extension at $w$ is. Lastly, I follow Hamblin (1971: 148), who suggests that the asker of a question and, in the case of acceptance, all the interlocutors are committed to the HP of the question.

Since possible answers are complete, a who-question like (7a) is taken as asking for the maximal plurality of individuals with the property of getting $100 \%$. After acceptance, the question becomes a common question in discourse and specifying this maximal plurality becomes a commitment of the interlocutors. Given this effect of (7a) on the context, the contrast between (7b) and (7c) arises because in (7c) the aree FC identifies the maximal plurality of individuals with the property of getting $100 \%$ with the entity denoted by the focused expression ${ }^{8}$ whereas no such identification necessarily exists in (7b). Put differently, (7b) says that Juan and Maria are two atoms of the maximal plurality of individuals with the property of getting $100 \%$ whereas (7c) says that that maximal plurality is the plurality with the two atoms Juan and Maria. Therefore, it is infelicitous in (7c) to continue by saying that one doesn't know whether there are other individuals that belong to this plurality or that this plurality contains another atom, namely the one for Juana, whereas no such infelicity arises in the case of (7b). In sum, the so-called exhaustivity implication is derivable from the two properties of the aree FC when it is used as an answer: (i) it takes as antecedent the maximal plurality of individuals with the property in question, which in turn is supplied by the meaning of the question, and (ii) it identifies this plurality with the denotation of the focused expression. I will, therefore, not analyze this implication as part of the meaning of the aree FC.

7 But, certainly, pragmatics doesn't always require answers to be complete.

8 This is similar to É. Kiss's (2006) claim that the Hungarian preverbal focus position is a predicative position and the exhaustivity is a result of specificational predication. 
To further illustrate the properties of the aree FC, consider the data below which show that the way that aree FCs identify the maximal plurality of individuals with the property in question might not always be as fine-grained as giving names. In $(8 b, c)$, the speaker provides coarse-grained information (Ginzburg 1995) to the best of his knowledge about the maximal plurality with the property in question.

(8) Context: Maria comes back from the kitchen and sees that the last tamale she had left on the table is gone. She asks $(8 a) .(8 b, c)$ are possible answers.

a. Chin $x-\emptyset-u-k$ 'am b'i k'isb'al sub'? who CMP-A3-E3-take DIR last tamale 'Who took the last tamale?'

b. Aree a Raul, aree al Maria ema k-na'taj=t chi-w-e chin FOC CLF Raul FOC CLF Maria but ICP-remember=NEG PREP-E1-GEN who chi-k-e. PREP-E3P-GEN

'It was Raul or Maria but I don't remember which.'

c. Aree jun chi-k-e ri ak'al-ab' ema k-na'taj=t chi-w-e FOC One PREP-E3P-GEN DET child-PL but ICP-remember=NEG PREP-E1-GEN chin chi-k-e. who PREP-E3P-GEN

'It was one of the kids but I don't remember which.'

In (9b), the speaker is providing information about the maximal plurality, identifying it with the property of being somebody that the addressee doesn't know.

(9) Context: Raul hears footsteps out in the yard. He later asks Roberto, who was outside, (9a). (9b) is a possible answer.

a. Chin $x-\emptyset$-pet-ik?

who CMP-A3-come-ss

'Who came?'

b. Aree (k'o) jun che $\emptyset$-aw-eta'm=t a-wach. FOC exist one COMP A3-E2-know=NEG E2-face 'It was somebody that you don't know.'

In (10b) and (10c), the aree FC is not used to identify an antecedent by adding information but by revising (mis)information about it.

(10) Context: Raul and his friend Roberto are watching two cats (one black, one white) in their yard. Roberto says (10a). Raul says (10b) or (10c).

a. X- $\emptyset$-aw-il-o? Ri saq me's X- $\emptyset$-u-chap jun tz'ikin. CMP-A3-E2-see DET white cat CMP-A3-E3-catch one bird 'Did you see? The white cat caught a bird.' 
Two kinds of focus constructions in K'iche'

b. K- $\emptyset$-in-b'ij in che aree ri q'eq $\mathrm{x}-\emptyset$-u-chap-o. ICP-A3-E1-say I COMP FOC DET black CMP-A3-E3-catch-SS 'I think it's the black one that caught it.'

c. K- $\emptyset$-in-b'ij in che aree jun pepe $\mathrm{x}-\emptyset$-u-chap-o. ICP-A3-E1-say I COMP FOC one butterfly CMP-A3-E3-catch-SS 'I think it's a butterfly that it caught.'

Examples $(10 b, c)$ illustrate another point about the aree FC: its felicitous use does not require it to be an answer to a question, as further exemplified in (11). Note, furthermore, that the so-called "exhaustivity" does not arise in such cases: the construction simply picks up an antecedent to provide more information about it but the antecedent itself isn't entailed to be maximal with respect to some property. This shows that exhaustivity only arises in the case of questions like (7a) where an aree FC is used as an answer since in such cases it will be identifying the maximal plurality of individuals with the property in question.

(11) Context: Maria and Juana are talking about their friend Raul who recently went to the nearby town. Maria says (11a) and Juana says (11b).

a. A Raul x- $\emptyset$-u-b'ij che $\mathrm{x}-\emptyset$-u-k'am lo jun sipanik ch-aw-e. CLF Raul CMP-A3-E3-say COMP CMP-A3-E3-take DIR one gift PREP-E3-GEN 'Raul said that he brought you a gift.'

b. Jee', aree kab'/ jun puwi' (x-Ø-u-k'am lo-q).

yes FOC candy one hat CMP-A3-E3-take DIR-SS

'Yes, it was candy/ a hat (that he brought).'

To conclude this section, I will discuss two claims from Can Pixabaj \& England 2011. As I mentioned above, Can Pixabaj \& England (2011: 21) claim that aree FCs have a cleft-like meaning and classify them under the heading of "contrastive" focus. They also seem to imply that aree can only be used with definite nominals. But as we've seen in (8b), (9), (10c) and (11b) such a requirement does not exist. Second, they claim that "contrastive" focus constructions require either aree "FOC' or xow 'only' preceding the focused expression. As I will discuss in detail in $§ 5$, most of the literature on constructions similar to the aree FC, e.g., it-clefts in English or Hungarian preverbal focus, claimed that their interpretations involve an only-type meaning. However, aree and the translational equivalent of only in K'iche' are not interchangeable as (12) shows. ${ }^{9}$

(12) Context: Raul is a new teacher at the primary school. He knows the people in the village but doesn't yet know who the parents of each of his students are. At a meeting, he asks Roberto, his fellow teacher, (12a) and Roberto says (12b).

9 The consultants I worked with used the variant xiw 'only' instead of xow. 
a. Chin u-tat al Maria?

who E3-father CLF Maria

'Who is Maria's father?'

b.\#Xiw/ Aree Carlos.

only FOC Carlos

'Only/ It is Carlos.'

In the next section, I turn to a formal account that captures the empirical generalizations I established so far.

\section{Formal analysis}

In this section, I sketch a dynamic analysis of the two focus constructions in K'iche' that captures the ideas developed in $\S 3$. I couch this analysis in a dynamic framework along the lines of Kierstead \& Martin (2012) and Martin (2013). This work builds on the line of work in dynamic semantics (e.g., Muskens 1996; Beaver 2001; de Groote 2006) that uses higher-order logic (HOL) to express the semantics. The framework I will be using is called Dynamic Categorial Grammar (DyCG, Martin 2013) which interfaces with a categorial grammar formalism called Linear Categorial Grammar (LCG, Pollard \& Smith 2012; Mihaliček 2012; Pollard 2013), which in turn is inspired by Oehrle (1994). The underlying semantic theory used in DyCG is written in HOL with the addition of the basic (non-logical) types e for entities, $\mathrm{p}$ for propositions and $\mathrm{w}$ for worlds. From these basic types, complex types that can be meanings of linguistic expressions are defined by the type constructor $\rightarrow$, e.g., e $\rightarrow$ $\mathrm{p}$ and $\mathrm{e} \rightarrow \mathrm{e} \rightarrow \mathrm{p}$ for unary and binary (static) properties, respectively.

DyCG models discourse contexts as functions from $n$-tuples of entities to propositions. Such a function, which is called an $n$-ary context, has the type $\mathrm{c}_{n}$ defined in (13) and would yield a proposition when applied to an $n$-tuple of entities.

(13) $\mathrm{c}_{n}=\mathrm{e}^{n} \rightarrow \mathrm{p}$

Intuitively, the $n$ components of the tuple correspond to the $n$ discourse referents (DRs) that the context "knows about." Vector notation is used when referring to tuples of DRs, e.g., $\mathbf{x}$ : $\mathrm{e}^{n}$ is an $n$-tuple of entities and the $i$-th component of $\mathbf{x}$, written $x_{i}$, corresponds to the $i$-th DR. As shorthand, I sometimes use $\mathbf{x}^{n}$ to indicate that $\mathbf{x}$ is of type $\mathrm{e}^{n}$. DyCG follows the dynamic tradition (e.g., Heim 1983) in treating the meaning of utterances of declarative sentences as functions from contexts to contexts. To illustrate, the dynamic content that the sentence $A$ cat chased a mouse contributes is given in (14a) where the propositional constant and is of type $\mathrm{p} \rightarrow$ $\mathrm{p} \rightarrow \mathrm{p}$. This content takes a context $c$, whose DRs are notated as $\mathbf{y}^{|c|}$ where $|c|$ 
is the number of DRs in $c$, and adds two new DRs to it. ${ }^{10}$ The acceptance of this utterance results in the update function given in (14b) where the first conjunct is the 'carryover' from whatever will be the input context, and the rest arises from the content of (14a). If the input context is "out-of-the-blue", then this update would yield the 2-ary context in (14c).
a. $\lambda_{c} \cdot \lambda_{\mathbf{y}^{|c|}, \mathbf{x}^{2}} \cdot\left(\right.$ cat $\left.x_{0}\right)$ and (mouse $\left.x_{1}\right)$ and (chase $\left.x_{1} x_{0}\right)$
b. $\lambda_{c} \cdot \lambda_{\mathbf{y}}|c|, \mathbf{x}^{2} \cdot(c \mathbf{y})$ and (cat $\left.x_{0}\right)$ and (mouse $\left.x_{1}\right)$ and (chase $\left.x_{1} x_{0}\right)$
c. $\lambda_{\mathbf{x}^{2}} \cdot\left(\right.$ cat $\left.x_{0}\right)$ and (mouse $\left.x_{1}\right)$ and (chase $\left.x_{1} x_{0}\right)$

I'll make two additions to this dynamic setup to handle questions. First, I adopt a dynamic rendering of the Hamblin presumption which was discussed in $\S 3.2$. Recall that the Hamblin presumption (HP) of a question is the proposition that exactly one of the alternatives in the question denotation is true which, in static terms, amounts to (15). Here, $q: \mathrm{p} \rightarrow \mathrm{p}$, namely, a propositional property, is the type of questions, following Hamblin (1957).

(15) ham $=_{\text {def }} \lambda_{q}$. exists! $p .(q p)$ and $p$

The second addition involves the representation of questions in a QUD-stack (Ginzburg 1994; Roberts 1996/2012) analyzed as a string of questions. Contexts become functions from a vector of entities to an ordered pair whose first component is a proposition (CG) and whose second component is a stack of questions (QUDstack) as in (16). Thus, (14c) is now represented as (17) where the second component corresponds to the empty QUD-stack.

(16) $\mathrm{c}_{n}==_{\text {def }} \mathrm{e}^{n} \rightarrow\left(\mathrm{p} \times \mathrm{q}^{*}\right)$

(17) $\lambda_{\mathbf{x}^{2}} \cdot\left\langle\left(\right.\right.$ cat $\left.x_{0}\right)$ and (mouse $\left.x_{1}\right)$ and (chase $\left.\left.x_{1} x_{0}\right),<>\right\rangle$

Now, assume that the question in (5), repeated below in (18), is uttered in a context $c$ where the existence implication of the aree FC is satisfied. Thus, both (18a) and (18b) are felicitous. Following Hamblin, the question is assigned the meaning in (19a). Here, I distinguish between types e (entities) and $\mathrm{e}^{\#}$ (pluralities); $X$ and $Y$ range over the latter. person ${ }_{\#}$ and $\mathrm{HAC}_{\#}$ are distributive properties of pluralities and $\mathrm{HAC}_{\#}$ abbreviates the property of having a computer. $Z$ ranges over properties of pluralities. (maximize $X Z$ ) is the proposition that the plurality $X$ is the maximal

10 I use the following notational conventions: lambda-bound variables are subscripted, e.g., I write $\lambda_{x}$. $a$ instead of $\lambda x$. $a$. I sometimes use a single $\lambda$ for multiple abstractions, e.g., $\lambda_{x y} . a$ instead of $\lambda x . \lambda y$. $a$. Object-language application is left-associative, hence (fab) abbreviates $((\mathrm{fa}) \mathrm{b})$. I eliminate outermost parentheses, so fab abbreviates $(f a b)$ and $f(a b)$ abbreviates $(f(a b))$. For any two vectors $\mathbf{x}$ and $\mathbf{y}, I$ write $\mathbf{x}, \mathbf{y}$ for their concatenation. 
plurality with property $Z$. Thus, the question is asking for the maximal plurality with the property of having a computer. The HP of the question is given in (19b) which is equivalent to (19c) where the uniqueness symbol ! is eliminated, since $X$ is unique.

(18) Context: Who has a computer?

a. A Raul (k'o jun u-kematz'ib'). b. Aree a Raul (k'o jun u-kematz'ib'). CLF Raul exist one E3-computer 'Raul (has a computer).' FOC CLF Raul exist one E3-computer 'It is Raul (who has a computer).'

a. who $\mathrm{HAC}_{\#}=\lambda_{p}$.exists $X \cdot p$ equals

$\left(\right.$ maximize $X\left(\lambda_{Y} \cdot\left(\right.\right.$ person $\left._{\#} Y\right)$ and $\left.\left.\left(\mathrm{HAC}_{\#} Y\right)\right)\right)$

b. exists! $p \cdot p$ and exists $X$. $(p$ equals

(maximize $X\left(\lambda_{Y} \cdot\left(\right.\right.$ person $\left._{\#} Y\right)$ and $\left.\left.\left.\left(\mathrm{HAC}_{\#} Y\right)\right)\right)\right)$

c. exists $X$. maximize $X\left(\lambda_{Y} \cdot\left(\right.\right.$ person $\left._{\#} Y\right)$ and $\left.\left(\operatorname{HAC}_{\#} Y\right)\right)$

Note that (19c), the static version of the HP of the question in (18), is a necessary truth since there has to be some (possibly null) plurality which is the maximal plurality with the property in question. What is of interest is the dynamic counterpart of $(19 \mathrm{c})$. Since the HP becomes a commitment of the discourse participants when a question is accepted, the acceptance of the question in $(18)^{11}$, and hence the addition of its HP to the CG, introduces the DR $X$ which is the maximal DR bearing the property in question. The acceptance of the question in (18) yields the update in (20) which has the effect of conjoining its HP to the input CG and pushing the question onto the QUD-stack.

$$
\begin{gathered}
\lambda_{c} \cdot \lambda_{\mathbf{y}|c|, X} \cdot\left\langle\left(\pi_{1}(c \mathbf{y})\right) \text { and }\left(\text { maximize } X\left(\lambda_{Y} \cdot\left(\text { person }_{\#} Y\right) \text { and }\left(\mathrm{HAC}_{\#} Y\right)\right)\right)\right. \\
\text { push (who HAC } \left.)\left(\pi_{2}(c \mathbf{y})\right)\right\rangle
\end{gathered}
$$

Given the above model, answers are analyzed such that they take as antecedent the DR that is introduced by the HP of the question. This way, the analysis captures the fact that answers are anaphoric to question continuations, that is, a functional abstraction of the non-wh part of a question. ${ }^{12}$ Depending on whether the focused expression in the answer is indefinite, definite or some other kind of generalized quantifier, we will have different cases. For instance, in the first case the corresponding DR will be newly introduced, in the second it will be one of the old ones, and in the third case a quantificational NP will be quantified into the focus position.

11 For simplicity, I assume that every question that gets asked is accepted. In a full-blown theory, we need to model how questions are accepted or rejected.

12 In fact, this analysis seems to make a QUD-stack unnecessary since the question continuation is already retrievable from the context. Rather, what we may need is to keep track of discourse topics. I will not develop this idea here but see Yasavul \& Pollard in prep for an analysis along those lines. 
To capture all of these compositionally is beyond the scope of this paper so I will confine myself to a sketch below (but see Yasavul \& Pollard in prep for an analysis). To capture a case like (18a), I define the function ans(wer) as in (21).

(21) The dynamic content of ans $x \mathrm{HAC}_{\#}$ :

a. is a function which takes as its argument a context $c$ with a nonempty QUD-stack where the topmost question is (who $\mathrm{HAC}_{\#}$ ),

b. asserts that the entity denoted by the focused expression is an atom of the maximal plurality of individuals bearing the property $\mathrm{HAC}_{\#}$.

As for an aree FC like (18b), recall that it takes as antecedent a DR with the relevant property and identifies this DR with the denotation of the focused expression. As discussed in $\S 3.2$, when there is a question that an aree $\mathrm{FC}$ is an answer to, the antecedent DR is made available via the HP of the question. When there is no question in the context, the relevant DR must be entailed to exist, either because it is introduced by an indefinite in prior discourse or because it is weakly familiar (Roberts 2003), that is, it is inferred to exist. The analysis below covers both of these cases.

(22) The dynamic content of aree $x \mathrm{HAC}_{\#}$ :

a. is a function that takes as its argument a context $c$ which entails that some DR has property HAC\#,

b. identifies that DR with property $\mathrm{HAC}_{\#}$ with the denotation of the focused expression.

Note that I haven't explicitly discussed how the anaphoric retrievability is captured in either of these constructions, which is why the above analyses are semiformal as they stand. In particular, it is possible to analyze anaphoric retrievability as a fundamentally pragmatic phenomenon where the way that the right antecedent DR is picked up wouldn't be built into the meaning of anaphoric triggers like the two focus constructions I discussed above. Especially important are cases like (18a,b) where the property in question is elided and thus the construction needs to be able to pick up the right DR without this property as argument. I leave it for future research to further develop this analysis.

Although the two focus constructions in K'iche' share certain similarities, e.g., both involve expressions in the pre-predicate focus position and both can be answers to questions, their meanings are quite different. Not only does the aree FC have an existence implication and give rise to an exhaustivity implication when it is used as an answer, but crucially the analysis of this construction cannot be built on that of the answer FCs (contra Bende-Farkas's 2006 claim for the corresponding case in Hungarian), as the former is not anaphoric to a question. However, what is common 
in the analyses of both of these constructions is that they employ continuations (see Barker 2002, 2004). Both of the expressions formed by applying ans and aree to their first arguments yield functors looking for the property that is obtained by abstracting on these expressions in the respective sentences, namely, their continuations. In other words, the denotation of both ans $x$ and aree $x$ are functions of their own continuations similar to the class of constructions analyzed under the heading of focus in the literature (Rooth 1992). This way of looking at focus constructions suggests that every answer to a question can be considered a focus construction and yet not every focus construction is an answer to a question.

In the next section, I discuss some prominent analyses of cleft/focus-movement constructions in order to compare them with the account I propose.

\section{Comparison with previous accounts}

The discussion in this section is restricted to the literature on it-clefts in English and preverbal focus in Hungarian, two much-discussed constructions which are similar to the aree FC in K'iche'. As mentioned in $\$ 4$, it is generally claimed that such constructions identify the focused expression as the only or the maximal entity with the relevant property, hence the so-called exhaustivity implication (among others, Horn 1981; Szabolcsi 1981; Roberts 1998; Percus 1997; É. Kiss 1998; Krifka 2008; Büring 2011; Velleman et al. 2012). For instance, it is argued that in (23a), when uttered in a context where the existence presupposition is satisfied, the cleft conveys that nobody other than John went to SALT or that John exhausts the list of SALT-goers, hence the infelicity of the sentences following the cleft in (23a).

(23) Context: Who went to SALT?

a. It was John. \#Bill went, too./ \#I don't know if anyone else did.

b. John. Bill went, too./ I don't know if anyone else did.

The exhaustivity implication was analyzed as an assertion by Bolinger (1972); Halvorsen (1978); Atlas \& Levinson (1981); Szabolcsi (1981); Han \& Hedberg (2008). However, Horn (1981) and, following him, Wedgewood (2007) argued that this analysis is problematic for English and Hungarian. In particular, if exhaustivity was asserted, then one wouldn't expect (24a) to be infelicitous but rather pattern like (24b), because the cleft would be informative (examples adapted from Horn 1981). ${ }^{13}$

(24) a.\#I know John went to SALT but I've just heard it was John who went to SALT.

b. I know John went to SALT but I've just heard only John went to SALT.

13 As I alluded to above, the common assumption seems to be that the exhaustivity implication of a cleft is somehow on a par with that of an exclusive like only. More on this below. 
Two kinds of focus constructions in K'iche'

Horn (1981) claimed that the exhaustivity implication should rather be analyzed as a conversational implicature. In his analysis, Horn argues that the existence presupposition of a cleft, together with what a cleft asserts, gives rise to the implication that the individual denoted by the focused expression is the only relevant individual with the property under discussion. According to Horn, the exhaustivity implication follows from a quantity implicature because if there were other individuals with the same property, the speaker would have mentioned them. The problem with this analysis, as Velleman et al. (2012) note, is that this implicature cannot be directly canceled as shown in (25).

(25)\#It was John who went to SALT; indeed, it was John and Bill.

A third line of analysis claims that the exhaustivity implication is a presupposition (Kenesei 1986; Szabolcsi 1994; Percus 1997; Bende-Farkas 2006; Hedberg 2013). In some presuppositional analyses, e.g., Percus 1997; Bende-Farkas 2006; Hedberg 2013, the similarities between cleft-like structures and definite descriptions are exploited, building on the idea that clefts conceal a discontinuous definite description, and equating the exhaustivity implication with the uniqueness (or maximality) presupposition of this definite description. In some of these analyses, the exhaustivity implication of a cleft like (26) is captured by employing a universal quantifier as in (26a) and in yet others it is captured by the iota operator as in (26b).

(26) It was John who went to SALT.
a. $\forall x$ (went-to-SALT $(x) \rightarrow(x=\mathrm{j}))$
c. $\exists x$ went-to-SALT $(x)$
b. $\mathrm{j}=\imath x$ went-to-SALT $(x)$
d. went-to-SALT (j)

Velleman et al. (2012) point out that the analyses of exhaustivity that employ (26a) are problematic because taken together with an existence presupposition like (26c), they entail (26d), the implication which is taken to be the asserted content. ${ }^{14}$ Consequently, the use of a cleft is predicted to be uninformative in contexts where these presuppositions are part of the CG or are accommodated, despite the fact that clefts do not sound redundant in such contexts. Moreover, (26a) does not seem to survive under negation as examples like (27) are perfectly fine.

(27) It wasn't John who went to SALT. It was Bill.

As mentioned above, the interpretation of exhaustivity in clefts/focus-movement is usually taken to involve a counterpart of only. In the literature on Hungarian, too, it has sometimes been claimed that the analysis of preverbal focus involves an operator which is a silent counterpart of only. This covert exhaustivity operator is

14 Note that in the analysis I propose, the asserted content would not be (26d) but rather something along the lines of (26b). 
claimed to be responsible for the "exhaustive-identification" property of Hungarian preverbal focus as well as the syntactic movement to this position (Horvath 2000). Velleman et al. (2012: 442) note that the idea that clefts and only-sentences are semantically similar has resulted in analyses where the two are claimed to have the same exhaustive meaning component. The difference, as they note, was captured by claiming that only-sentences assert, while clefts presuppose or conventionally implicate, exhaustivity.

Velleman et al. (2012: 442) propose a similar account where they describe itclefts as "inquiry terminating (IT) constructions". An IT-construction is claimed to mark an answer to the current question under discussion as a maximal answer and, as a result, to resolve the question and terminate it as an active line of inquiry. In Velleman et al.'s analysis, both only and $\mathrm{CLEFT}_{S}$, their cleft operator responsible for the exhaustivity implication of a cleft, are characterized by using the two meaning components MIN and MAX given in (28). Here, the subscript $S$ indexes the current context which includes a Current Question under discussion ${ }^{15}\left(C Q_{S}\right)$ and a salient partial ordering over the alternatives in $C Q_{S}$, indicated by the entailment relations ${ }^{16}$ $\left(\geq_{S}\right)$ and $\left(>_{S}\right)$. In particular, $p \geq_{S} q$ iff $p$ entails $q$, and $p>_{S} q$ iff $p$ entails $q$ and $p$ is distinct from $q$.

a. $\operatorname{MIN}_{S}(p)=\lambda w \cdot \exists q \in C Q s[q(w) \wedge(q \geq s p)]$ "There's a true answer at least as strong as $p . "$

b. $\operatorname{MAX}_{S}(p)=\lambda w . \forall q \in C Q s\left[\left(q>_{S} p\right) \rightarrow \neg q(w)\right]$ "No true answer is strictly stronger than $p . " \quad$ (Velleman et al. 2012: 451)

According to Velleman et al., clefts and only-sentences differ in their meaning because what is at-issue for $\mathrm{CLEFT}_{S}$, namely, (28a), is presupposed for only, and what is at-issue for only, namely, (28b), is presupposed for CLEFT $S$. Consequently, they assign (29a) to only and (29b) to CLEFT $S$. The two meaning components are separated by a dot as follows: presupposed.asserted. Given (29b), Velleman et al. would derive the exhaustivity in (26) as follows. The MIN component asserts that there's a true answer at least as strong as (26d), and the MAX component requires that there is no answer stronger than (26d), and as a result one infers that John went to SALT and nobody other than John did.

a. $\llbracket$ only $\rrbracket=\lambda w \cdot \lambda p: \operatorname{MIN}_{S}(p)(w) \cdot \operatorname{MAX}_{S}(p)(w)$

b. $\operatorname{CLEFT}_{S}=\lambda w \cdot \lambda p: \operatorname{MAX}_{S}(p)(w) \cdot \operatorname{MIN}_{S}(p)(w)($ Velleman et al. 2012: 452)

The Velleman et al. analysis has several problems, though. First, an it-cleft doesn't have to resolve a question and terminate it as an inquiry. For example,

15 The term Current Question (CQ) is used to refer to the question at the top of the QUD-stack in the sense of Roberts 1996/2012.

16 These relations may include pragmatic ordering alongside pure entailment (Beaver \& Clark 2008). 
depending on the goals of the querier in (30), the clefts in (30a-c) may be taken as identifying "the cookie-taker" to the best of the cleft-utterer's knowledge but this may not sufficiently answer the question for it to be popped out of the QUD-stack.

(30) Who took the last cookie?

a. It wasn't one of the kids.

b. It was one of the kids but I don't remember which.

c. It was John or Mary but I don't remember which.

Velleman et al. (2012: 449) also claim that it-clefts require a question to have been raised or discussed. They propose that the existence presupposition of a cleft is triggered by the question it answers (Velleman et al. 2012: 456). However, an it-cleft in English, just as the aree FC in K'iche' (see (10) and (11) above), doesn't require a question to be under discussion in prior discourse. In (31), ${ }^{17}$ for example, there's nothing in the input context that gets the question Who robbed the BP station? onto the QUD-stack. Rather, in (31a), the speaker B chooses an antecedent (a weakly familiar DR), namely the one for the robber(s), since the existence of such a DR is inferable from the existence of a robbery, and equates this DR with the denotation of the post-copular NP. The fact that (31b) is infelicitous in this context casts further doubt that the cleft in (31a) is an answer to a question. In (31c), the speaker B chooses another DR, namely the one for the gas station (a strongly familiar DR). Similar to the aree FC, an exhaustivity inference does not arise because the CG does not entail that the antecedent DRs are maximal with respect to some property; the cleft just picks up an antecedent DR to further identify it.

(31) A: The gas station near Bethel and River Road was robbed last night!

B: a. It was my two halfwit brothers (who robbed it).

b. \#My two halfwit brothers.

c. It must be the BP station (that was robbed).

As for the meaning in (29b) that is assigned to clefts, note that $\operatorname{MIN}_{S}(p)$ is actually $p^{18}$ so in (26), for example, the asserted content would be (26d) which is the same as what most of the previous accounts claimed it to be. On the other hand, $\operatorname{MAX}_{S}(p)$ will be truth-conditionally equivalent to the proposition If John went to SALT, then nobody else did. This is the same conditional presupposition that Burring (2011) proposed for it-clefts except that Velleman et al. claim that the meaning of clefts are focus sensitive in that they make reference to the current QUD. Either way,

17 This example is due to Carl Pollard.

18 Velleman et al. note that $p \models \operatorname{MIN}_{S}(p)$, in other words $p \subseteq \operatorname{MiN}_{S}(p)$. The fact that $\operatorname{MiN}_{S}(p) \subseteq p$ follows from the definition of $\operatorname{MIN}_{S}(p)$ and the fact that worlds are closed under entailment. 
such a presupposition seems unnecessary. For instance, for (32a) to be felicitous, it is not necessary neither for the speaker to assume (32b) to be mutually known, nor for the hearer to know or to accommodate it.

(32) Who came to see you last night?

a. It was somebody that you don't know.

b. ps: (?) If somebody that the addressee doesn't know came, then nobody else did.

Lastly, a Velleman et al.-style exhaustivity account cannot be extended to cases like (30c). To say that (30c) presupposes that no true answer is strictly stronger than the proposition (took-last-cookie $(\mathrm{j}) \vee$ took-last-cookie $(\mathrm{m})$ ) seems false because (30c) implicates that there $i s$ a true answer which is strictly stronger than this disjunction depending on who actually took the last cookie.

The analysis I developed in $\$ 4$ does not have any of these problems. For one thing, I argued that the existence presupposition has to be satisfied independently of a question. In cases like (31), given what A says, the antecedent DR is either weakly familiar or strongly familiar, satisfying the existential presupposition in each case. As for cases like (30) and (32), we can simply say that the use of the cleft provides more information about the antecedent DR.

\section{Conclusion}

In this paper, I claimed that the two focus constructions in K'iche' have different interpretations, a distinction which has been overlooked or not explicitly shown in the previous literature. In particular, I argued that the interpretation of the aree FCs gives rise to existence and exhaustivity implications (the latter when they are used as answers) both of which do not necessarily arise from the interpretation of the answer FCs. This finding goes against the standard assumption that focus constructions in Mayan are interpreted like clefts across-the-board (Aissen 1992).

I concentrated on the proper analysis of the implications of the aree FCs and reviewed the previous accounts proposed for similar constructions like English itclefts and Hungarian preverbal focus. I argued that an exhaustivity implication is not part of the meaning of the aree FCs as it only arises when this construction is used as an answer to, say, a who-question where the antecedent that the aree FC picks up is entailed to be maximal due to the meaning of the question. I also sketched a dynamic analysis that captures the differences between the two constructions. Finally, I argued that despite certain similarities, the two focus constructions in K'iche' receive different analyses and, crucially, that the analysis of the aree FCs cannot be built on that of the answer FCs since the former are not anaphoric to questions. 
Two kinds of focus constructions in K'iche'

\section{References}

Aissen, Judith. 1992. Topic and focus in Mayan. Language 68(1). 43-80.

Atlas, Jay David \& Stephen C. Levinson. 1981. It-clefts, informativeness and logical form: radical pragmatics. In Peter Cole (ed.), Radical Pragmatics, 1-61. New York: Academic Press.

Barker, Chris. 2002. Continuations and nature of quantification. Natural Language Semantics 10. 211-242.

Barker, Chris. 2004. Continuations in natural language. In H. Thielecke (ed.), Fourth ACM SIGPLAN Continuations Workshop (CW'04)., 1-11. Technical Report CSR-04-1, School of Computer Science, University of Birmingham, Birmingham B15 2TT, United Kingdom.

Beaver, David. 2001. Presupposition and Assertion in Dynamic Semantics. CSLI Publications.

Beaver, David I. \& Brady Z. Clark. 2008. Sense and Sensitivity: How Focus Determines Meaning. Oxford: Wiley-Blackwell.

Bende-Farkas, Ágnes. 2006. Comparing English and Hungarian focus. Ms, IMS Stuttgart University.

Bolinger, Dwight. 1972. A look at equations and cleft sentences. In Evelyn Firchow (ed.), Studies for Einar Haugen, 96-114. The Hague: Mouton de Gruyter.

Büring, Daniel. 2011. Conditional exhaustivity presuppositions in clefts (and definites). Ms. submitted.

Can Pixabaj, Telma \& Nora England. 2011. Nominal topic and focus in K'ichee'. In Rodrigo Guitérrez-Bravo, Line Mikkelsen \& Eric Potsdam (eds.), Representing Language: Essays in Honor of Judith Aissen, 15-30. Linguistics Research Center, UC Santa Cruz Department of Linguistics.

Chierchia, Gennaro \& Sally McConnell-Ginet. 1990. Meaning and Grammar: An Introduction to Semantics. Cambridge, MA.: MIT Press.

Delin, J. 1992. Properties of it-cleft presupposition. Journal of Semantics 9. 179-196.

É. Kiss, Katalin. 1998. Identificational focus versus information focus. Language 74. 245-273.

É. Kiss, Katalin. 2006. Focussing as predication. In Valéria Molnár \& Susanne Winkler (eds.), Architecture of Focus, 169-196. Berlin: Mouton de Gruyter.

England, Nora. 1991. Changes in basic word order in Mayan languages. International Journal of American Linguistics 57(4). 446-486.

Ginzburg, Jonathan. 1994. An update semantics for dialogue. In Harry Bunt, Reinhard Muskens \& Gerrit Rentier (eds.), The Tilburg International Workshop on Computational Semantics, ITK, Tilburg.

Ginzburg, Jonathan. 1995. Resolving questions, I. Linguistics and Philosophy 18(5). 459-527. 
de Groote, Philippe. 2006. Towards a Montagovian account of dynamics. In Masayuki Gibson \& Jonathan Howell (eds.), Semantics and Linguistic Theory (SALT) 16, 1-16.

Halvorsen, Per-Kristian. 1978. The syntax and semantics of cleft constructions: University of Texas, Austin $\mathrm{PhD}$ dissertation.

Hamblin, Charles Leonard. 1957. Language and the theory of information: Logic and Scientific Method Programme, University of London PhD dissertation.

Hamblin, Charles Leonard. 1971. Mathematical models of dialogue. Theoria 2. 131-155.

Han, Chung-hye \& Nancy Hedberg. 2008. Syntax and the semantics of it-clefts: a Tree-Adjoining Grammar analysis. Journal of Semantics 25. 345-380.

Hedberg, Nancy. 2013. Multiple focus and cleft sentences. In Katharina Hartmann \& Tonjes Veenstra (eds.), Cleft Structures, 227-250. John Benjamins, Linguistik Aktuell series.

Heim, Irene. 1983. On the projection problem of presuppositions. In Michael Barlow, Dan Flickinger \& Michael Wescoat (eds.), West Coast Conference on Formal Linguistics (WCCFL) 2 114-225, Stanford: Stanford University.

Horn, Laurence R. 1981. Exhaustiveness and the semantics of clefts. In Victoria Burke \& James Pustejovsky (eds.), North East Linguistic Society (NELS) 11, 124-142. Amherst, GLSA.

Horvath, Julia. 2000. Interfaces vs the computational system in the syntax of focus. In Hans Bennis, Martin Everaert \& Eric Reuland (eds.), Interface Strategies, 183-206. Holland Academic Graphics.

Kenesei, Istvan. 1986. On the logic of word order in Hungarian. In Werner Abraham, Werner Abraham \& Sjaak de Meij (eds.), Topic, Focus, and Configurationality, 143-159. Amsterdam: John Amsterdam: John Benjamins.

Kierstead, Gregory \& Scott Martin. 2012. A multistratal account of the projective Tagalog evidential 'daw'. In Anca Chereches (ed.), Semantics and Linguistic Theory (SALT) 22, 326-346.

Krifka, Manfred. 2008. Basic notions of information structure. Acta Linguistica Hungarica 55. 243-276.

Larsen, Thomas W. 1988. Manifestations of ergativity in Quiché grammar: University of California, Berkeley PhD dissertation.

Martin, Scott. 2013. The dynamics of sense and implicature: Ohio State University $\mathrm{PhD}$ dissertation.

Matthewson, Lisa. 2004. On the methodology of semantic fieldwork. International Journal of American Linguistics 70(4). 369-415.

Mihaliček, Vedrana. 2012. Serbo-Croatian word order: a logical approach: Ohio State University $\mathrm{PhD}$ dissertation.

Muskens, Reinhard. 1996. Combining Montague semantics and discourse represen- 
Two kinds of focus constructions in K'iche'

tation theory. Linguistics and Philosophy 19(2). 143-186.

Oehrle, Richard T. 1994. Term-labeled categorial type systems. Linguistics and Philosophy 17(6). 633-678.

Percus, Orin. 1997. Prying open the cleft. In Kiyomi Kusumoto (ed.), North East Linguistic Society (NELS) 26 Amherst: GLSA, 337-351.

Pollard, Carl. 2013. Agnostic hyperintensional semantics. Synthese 1-28.

Pollard, Carl \& E. Allyn Smith. 2012. A unified analysis of the same, phrasal comparatives, and superlatives. In Anca Chereches (ed.), Semantics and Linguistic Theory (SALT) 22, 307-325.

Richards, Michael. 2003. Atlas Lingüistico de Guatemala. Guatemala City: Universidad de Rafael Landívar.

Roberts, Craige. 1996/2012. Information structure: towards an integrated formal theory of pragmatics. In Jae Hak Yoon \& Andreas Kathol (eds.), Ohio State University Working Papers in Linguistics (OSUWPL), vol. 49, 91-136. Ohio State University, Department of Linguistics. Reprinted with a new Afterword in Semantics and Pragmatics volume 5, 2012.

Roberts, Craige. 1998. Focus, the flow of information, and Universal Grammar. In Peter Culicover \& Louise McNally (eds.), The Limits of Syntax, 109-160. New York: Academic Press.

Roberts, Craige. 2003. Uniqueness in definite noun phrases. Linguistics and Philosophy 26. 287-350.

Rooth, Mats. 1992. A theory of focus interpretation. Natural Language Semantics 1(1). 75-116.

Szabolcsi, Anna. 1981. Compositionality in focus. Folia Linguistica Societatis Linguisticae Europeae 15. 141-162.

Szabolcsi, Anna. 1994. All quantifiers are not equal: The case of focus. Acta Linguistica Hungarica 42. 171-187.

Tonhauser, Judith, David Beaver, Craige Roberts \& Mandy Simons. 2013. Towards a taxonomy of projective content. Language 89(1). 66-109.

Trechsel, Frank R. 1993. Quiché focus constructions. Lingua 91(1). 33-78.

Velleman, Dan, David Beaver, Emilie Destruel, Dylan Bumford, Edgar Onea \& Liz Coppock. 2012. It-clefts are IT (inquiry terminating) constructions. In Anca Chereches (ed.), Semantics and Linguistic Theory (SALT) 22, 441-460.

Wedgewood, Daniel. 2007. Identifying inferences in focus. In Kerstin Schwabe \& Susanne Winkler (eds.), On Information Structure, Meaning, and Form, 207-227. Amsterdam \& Philadelphia: John Benjamins.

Yasavul, Murat \& Carl Pollard. in prep. A Hamblinian dynamic analysis of questions and answers. 
Yasavul

Murat Yasavul

204 Oxley Hall

The Ohio State University

Columbus, OH 43210 USA

murat@ling.osu.edu 